\title{
Robotic Assistance in Coordination of Patient Care
}

\author{
Matthew Gombolay*, Xi Jessie Yang*, Brad Hayes*, Nicole Seo*, Zixi Liu*, Samir Wadhwania*, Tania Yu*, \\ Neel Shah ${ }^{\dagger}$, Toni Golen ${ }^{\dagger}, \&$ Julie Shah* \\ * Massachusetts Institute of Technology, 77 Massachusetts Avenue, Cambridge, Massachusetts 02139 \\ ${ }^{\dagger}$ Beth Israel Deaconess Medical Center, 330 Brookline Avenue, Boston, Massachusetts 02215 \\ \{gombolay,xjyang\}@csail.mit.edu, \{hayesbh,nicseo,zixiliu,samirw,taniayu\}@mit.edu \\ \{ntshah,tgolen\}@bidmc.harvard.edu, and julie_a_shah@csail.mit.edu
}

\begin{abstract}
We conducted a study to investigate trust in and dependence upon robotic decision support among nurses and doctors on a labor and delivery floor. There is evidence that suggestions provided by embodied agents engender inappropriate degrees of trust and reliance among humans. This concern is a critical barrier that must be addressed before fielding intelligent hospital service robots that take initiative to coordinate patient care. Our experiment was conducted with nurses and physicians, and evaluated the subjects' levels of trust in and dependence on high- and low-quality recommendations issued by robotic versus computer-based decision support. The support, generated through action-driven learning from expert demonstration, was shown to produce high-quality recommendations that were accepted by nurses and physicians at a compliance rate of $90 \%$. Rates of Type I and Type II errors were comparable between robotic and computer-based decision support. Furthermore, embodiment appeared to benefit performance, as indicated by a higher degree of appropriate dependence after the quality of recommendations changed over the course of the experiment. These results support the notion that a robotic assistant may be able to safely and effectively assist in patient care. Finally, we conducted a pilot demonstration in which a robot assisted resource nurses on a labor and delivery floor at a tertiary care center.
\end{abstract}

\section{INTRODUCTION}

Service robots are being increasingly utilized across a wide spectrum of clinical settings. They are deployed to improve operational efficiency by delivering and preparing supplies, materials and medications [6, 15, 20, 35, 38]. The systems exhibit robust, autonomous capabilities for navigating from point to point while avoiding obstacles [33, 34], and initial concerns regarding physical safety around people have largely been addressed. However, these robots are not yet wellintegrated into the healthcare delivery process - they do not operate with an understanding of patient status and needs, and must be explicitly tasked and scheduled. This can impose a substantial burden upon the nurse in charge of resource allocation, or the "resource nurse," - particularly within fastpaced hospital departments, such as the emergency or labor and delivery units.

Resource nurses are essentially solving an NP-hard [5] problem on-the-fly: They assign resources such as beds (e.g. for triage, in-patient, recovery and operating rooms) while subject to upper- and lower-bound temporal constraints on

This work was supported by the National Science Foundation Graduate Research Fellowship Program under grant number 2388357, CRICO Harvard Risk Management Foundation, and Aldebaran Robotics Inc. availability and considering stochasticity in the timing of patient progression from one bed type to another. They must also pair patients with staff nurses, equipment and resources. The resource nurse's job is made feasible because staff nurses understand patients statuses and needs and will take initiative to accomplish some tasks without being explicitly directed.

As the number and types of hospital service robots increases, these robots must similarly take initiative in order to provide a net productivity benefit. The need to explicitly task many service robots may degrade the performance of a resource nurse [9, 11, 37], which has implications for both patient safety and the well-being of healthcare professionals [7, 24, 42, 47].

On the other hand, a robot that autonomously takes initiative when performing tasks may make poor decisions in the absence of oversight. Furthermore, decades of research in human factors cautions against fully autonomous decision making, as it contributes to poor human situational awareness and degradation in the human supervisor's performance [23, 41, 46, 53]. When integrating machines into human cognitive workflows, an intermediate level of autonomy is preferred [23, 53], in which the system provides suggestions to be accepted or modified by a human supervisor. Such a system would fall within the "4-6" range on the 10-point scale of Sheridan's levels of automation [41].

In this paper, we investigate the human factors implications of fielding hospital service robots that will necessarily reason about which tasks to perform and when to perform them. In particular, we investigate trust in and dependence upon robotic decision support among nurses and doctors on a labor and delivery floor. Studies of human-automation interaction in aviation - another safety-critical domain - have shown that human supervisors can inappropriately trust in and rely upon recommendations made by automation systems [16]. For example, numerous aviation incidents have been attributed to human overreliance on imperfect automation [16]. Other studies have examined the effects of changes in system reliability, and found that it led to suboptimal control allocation strategies and reduced levels of trust in the systems [13, 14]. There is also evidence that suggestions provided by embodied agents engender over-trust and inappropriate reliance [44]. This concern is a critical barrier to fielding intelligent hospital service robots that take initiative to participate with nurses in decision making. 


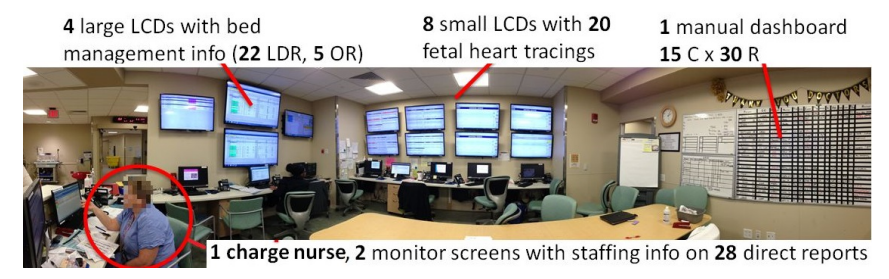

Fig. 1. A resource nurse must assimilate a large variety and volume of information to effectively reason about resource management for patient care.

This paper presents three novel contributions to the fields of robotics and healthcare. First, through human subject experimentation with physicians and registered nurses, we conducted the first known study involving experts working with an embodied robot on a real-world, complex decision making task comparing trust in and dependence on robotic versus computer-based decision support. Previous studies have focused on novice users and/or simple laboratory decision tasks [4, 12, 26, 31]. Our findings provide the first evidence that experts performing decision making tasks appear to be less susceptible to the negative effects of support embodiment, as trust assessments were similar in both the computer-based and robotic decision support conditions. Furthermore, embodiment yielded performance gains compared with computer-based support after the quality of recommendations changed over the course of the experiment. This provides encouraging evidence that intelligent service robots can be safely integrated into the hospital setting.

Second, decision support generated through action-driven learning from expert demonstration was shown to produce high-quality recommendations accepted by nurses and physicians at a compliance rate of $90 \%$. This indicates that a hospital service robot may be able to learn context-specific decision strategies and apply them to make reasonable suggestions for which tasks to perform and when.

Finally, based on the previous two findings, we conducted the first test demonstration in which a robot assisted resource nurses on a labor and delivery floor in a tertiary care center. Our robot used machine learning computer vision techniques to read the current status of the labor floor and make suggestions about resource allocation, and used speech recognition to receive feedback from the resource nurse. To our knowledge, this is the first investigation to field a robotic system in a hospital to aid in the coordination of resources required for patient care.

\section{BACKGROUND}

While the effects of embodiment on engagement in social judgment tasks are extensively studied and well-documented (e.g. [25, 26, 48, 49]), the relationship between embodiment and humans levels of trust and dependence is a relatively new area of research [4, 26, 31]. This topic is crucial if robots are to become more than companions, but advisors to people.

Trust is defined as "the attitude that an agent will help achieve an individual's goals in a situation characterized by uncertainty and vulnerability [28]," and dependence is a behavioral measure indicating the extent to which users accept the recommendation of robots or virtual agents. Measures of dependence are distinguished according to whether the user makes Type I or Type II errors [17]. "Type I" refers to reliance, or the degree to which users accept advice from an artificial agent when it offers low-quality recommendations. "Type II" refers to the extent to which human users reject advice from an artificial agent when the advice is of high quality. The degrees to which a user accepts high-quality advice and rejects low-quality advice are called "appropriate compliance" and "appropriate reliance," respectively.

Studies examining the effects of embodiment on trust and dependence necessarily include objective assessments of dependence and task performance in addition to subjective assessment of the users trust in the system [4, 12, 26, 31, 40]. Scassellati et. al. [4, 31] conducted a series of experiments to compare compliance rates when interacting with a physically embodied robot, a video of a robot and a disembodied voice. The tasks involved users receiving instructions to move objects to different locations, along with strategy advice for solving Sudoku-like puzzles. The authors found that embodiment was associated with a higher rate of compliance with advice provided by the robot, and suggested this indicated a greater level of human trust for an embodied robot. Similarly, Kiesler et. al. [26] found that participants consumed fewer calories after receiving health advice from a physically embodied robot, as compared with advice from a video of a robot or an on-screen animated virtual agent.

Studies in human factors and decision support indicate that increased anthropomorphism also affects user interactions. Pak et al. [40] evaluated how the anthropomorphic characteristics of decision support aids assisting subjects answering questions about diabetes influenced subjective trust and task performance. The results indicated that younger adults trusted the anthropomorphic decision aid more, whereas older adults were insensitive to the effects of anthropomorphism. Moreover, shorter question response time (after controlling for accuracy) was observed in both age groups, suggesting a performance gain when receiving advice from a more anthropomorphic aid. In another study, de Visser [12] varied the degree of anthropomorphism of a decision support system while participants performed a pattern recognition task. The results indicated that the perceived knowledgeableness of the system increased with increasing anthropomorphism; however, their findings on dependence were inconclusive.

The results from studies with embodied robots must be interpreted with caution since they were primarily focused on situations in which robots produced reliable and highquality recommendations. There is a growing body of research indicating that the quality of decision support cannot be relied upon, especially during complex tasks [52]. Negative consequences of humans blindly depending upon imperfect embodied artificial intelligence have been previously reported [44]. For example, Robinette et al. [44], conducted experiments in which a robot guided human participants during a mock emergency rescue scenario involving a building fire. All 
participants followed the robot, even when the robot led them down unsafe routes and/or displayed simulated malfunctions and other suspicious behavior.

Such dependence upon imperfect automation presents serious problems for robotic assistance during safety-critical tasks. This concern is heightened by results from studies indicating increased trust in and reliance upon embodied systems as compared with virtual or computer-based decision support, suggesting a higher possibility of committing Type I errors. However, we also note that prior studies on embodiment, trust and dependence were conducted with novices rather than domain experts performing complex real-world tasks. This leaves us with founded concerns, but gaps in our understanding of how human-robot interaction impacts the decision making of expert resource nurses. In the next sections, we describe our experiment and present a positive result for service robots in a hospital setting, with Type I and Type II error rates comparable to those observed for computer-based decision support. Furthermore, embodiment appeared to improve performance, as indicated by a higher degree of appropriate compliance when the quality of advice changed mid-experiment.

\section{EXPERIMENTAL INVESTIGATION}

In this section, we describe human-subject experimentation aimed at comparing trust in and dependence upon an embodied robot assistant versus computer-based decision support in a population of physicians and registered nurses. The participants interacted with a high-fidelity simulation of an obstetrics department at a tertiary care center. This simulation provided users the opportunity to assume the roles and responsibilities of a resource nurse, which included assigning labor nurses and scrub technicians to care for patients, as well as moving patients throughout various care facilities within the department.

We conducted the experiment using a within-subjects design that manipulated two independent variables: embodiment subjects received advice from either a robot or a computer, and recommendation quality - subjects received high- or lowquality advice. Each participant experienced four conditions, the quality of advice was blocked and the ordering of the conditions was counterbalanced in order to mitigate potential learning effects. Figure 2 depicts the experimental setup for the embodied condition.

\section{A. Hypotheses and Measures}

H1 Rates of appropriate compliance with and reliance on robotic decision support will be comparable to or greater than those observed for computer-based decision support. Objective measures of compliance and reliance were assessed based on the participants' "accept" or "reject" response to each decision support recommendation. Statistics on appropriate compliance, appropriate reliance, Type I and Type II errors were recorded. H2 Robotic decision support will be rated more favorably than computer-based decision support in terms of trust and other attitudinal measures. Numerous studies have demonstrated that embodied and anthropomorphic systems are rated

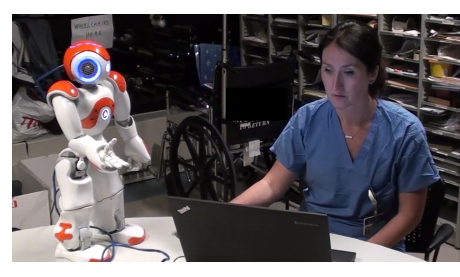

Fig. 2. Experiment participant pictured receiving advice from the robotic decision support.

more favorably by users than computer-based interactive systems. We hypothesized that the robotic system in our study would elicit this favorable response (H2), while engendering appropriate rates of compliance and reliance (H1). This would indicate a positive signal for the successful adoption of a hospital service robot that participates in decision making. Subjective measures of trust and attitudinal response were collected via questionnaires administered to each participant after each of the four trials. Trust was assessed by a composite rating of seven-point Likert-scale responses for a commonly used, validated trust questionnaire [21]. Other attitudinal questions were drawn from [29] to evaluate personality recognition, social responses and social presence in human-robot interaction, and were responded to on a 10-point Likert scale.

\section{B. Materials and Setup}

We conducted our experiments using a high-fidelity simulation of a labor and delivery floor. This simulation had previously been developed through a hospital quality improvement project as a training tool over a year-long, rigorous design and iteration process that included workshops with nurses, physicians, and medical students to ensure the tool accurately captured the role of a resource nurse. Parameters within the simulation (e.g. arrival of patients, timelines on progression through labor) were drawn from medical textbooks and papers and modified through alpha and beta testing to ensure that the simulation closely mirrored the patient population and nurse experience at our partner hospital.

An Aldebaran Nao was employed for the embodied condition (Figure 22. A video of the Nao offering advice to a participant with speech and co-speech gestures is viewable at http://tiny.cc/NAORecommendation. Participants received advice through synthesized speech under both the embodied and computer-based support conditions, using a male voice drawn from the Mary Text-to-Speech System (MaryTTS) [45]. The advice was also displayed as text in an in-simulation popup box under both conditions. The subject clicked a button in order to accept or reject the advice. These buttons were not clickable until the narration of the advice was complete; this narration took equal time in both conditions.

\section{Experimental Procedure}

Seventeen physicians and registered nurses participated in the experiment (one man and sixteen women). The participants were recruited from the partner hospital's obstetrics department via email and word-of-mouth. 
First, participants provided consent for the experiment and watched an 8-minute tutorial video describing the labor and delivery floor simulation. The tutorial video is viewable at http://tiny.cc/simTutorial Participants were instructed to play the simulation four times, with each iteration lasting 10 minutes, simulating a total of 4 hours on the labor floor. The computer or embodied system would interject during the simulation to make recommendations on which nurse should care for which patient, and on patient room assignments. Participants were asked to accept or reject the advice based on their own judgment. They were not informed whether the robotic or virtual decision support coach was providing highor low-quality advice. Finally, after each of the four trials, participants were asked to rate their subjective experience via a set of Likert-scale questions, as described in Section III-A

\section{TOWARD DECISION SUPPORT - Formulation OF THE Resource Nurse's Decision-Making Problem}

This section provides a formal representation of the resource nurse's decision making problem. Section $\mathrm{V}$ describes how we implemented the decision support based on this formulation.

A resource nurse must solve a problem of task allocation and schedule optimization with stochasticity in the number and types of patients and the duration of tasks. A task $\tau_{i}$ represents the set of steps required to care for patient $i$, and each $\tau_{i}^{j}$ is a given stage of labor for that patient. Stages of labor are related by stochastic lower-bound constraints $W_{\left\langle\tau_{i}^{j}, \tau_{x}^{y}\right\rangle}$, requiring the stages to progress sequentially. There are stochastic time constraints, $D_{\tau_{i}^{j}}^{a b s}$ and $D_{\left\langle\tau_{i}^{j}, \tau_{x}^{y}\right\rangle}^{r e l}$, relating the stages of labor to account for the inability of resource nurses to perfectly control when a patient will move from one stage of labor to the next. Arrivals of $\tau_{i}$ (i.e. patients) are drawn from stochastic distributions. The model considers three types of patients: scheduled cesarean patients, scheduled induction patients and unscheduled patients. The set of $W_{\left\langle\tau_{i}^{j}, \tau_{x}^{y}\right\rangle}, D_{\tau_{i}^{j}}^{a b s}$ and $D_{\left\langle\tau_{i}, \tau_{j}\right\rangle}^{r e l}$ are dependent upon patient type.

Labor nurses are modeled as agents with a finite capacity to process tasks in parallel, where each subtask requires a variable amount of this capacity. For example, a labor nurse may generally take care of a maximum of two patients. If the nurse is caring for a patient who is "fully and pushing" (i.e., the cervix is fully dilated and the patient is actively trying to push out the baby) or in the operating room, the nurse may only care for that patient.

Rooms on the labor floor (e.g., a labor room, an operating room, etc.) are modeled as resources, which process subtasks in series. Agent and resource assignments to subtasks are preemptable, meaning that the agent and resource assigned to care for any patient during any step in the care process may be changed over the course of executing that subtask.

In this formulation, ${ }^{t} A_{\tau_{i}^{j}}^{a} \in\{0,1\}$ is a binary decision variable for assigning agent $a$ to subtask $\tau_{i}^{j}$ for time epoch $[t, t+1) \cdot{ }^{t} G_{\tau_{i}^{j}}^{a}$ is an integer decision variable for assigning a certain portion of the effort of agent $a$ to subtask $\tau_{i}^{j}$ for time epoch $[t, t+1) \cdot{ }^{t} R_{\tau_{i}^{j}}^{r} \in\{0,1\}$ is a binary decision variable for whether subtask $\tau_{i}^{j}$ is assigned resource $r$ for time epoch $[t, t+1) . H_{\tau_{i}} \in\{0,1\}$ is a binary decision variable for whether task $\tau_{i}$ and its corresponding subtasks are to be completed. $U_{\tau_{i}^{j}}$ specifies the effort required from any agent to work on $\tau_{i}^{j^{i}} . s_{\tau_{i}^{j}}, f_{\tau_{i}^{j}} \in[0, \infty)$ are the start and finish times of $\tau_{i}^{j}$.

$$
\begin{gathered}
\min f n\left(\left\{{ }^{t} A_{\tau_{i}^{j}}^{a}\right\},\left\{{ }^{t} G_{\tau_{i}^{j}}^{a}\right\},\left\{{ }^{t} R_{\tau_{i}^{j}}^{r}\right\},\left\{H_{\tau_{i}}\right\},\left\{s_{\tau_{i}^{j}}, f_{\tau_{i}^{j}}\right\}\right) \\
\sum_{a \in A}{ }^{t} A_{\tau_{i}^{j}}^{a} \geq 1-M\left(1-H_{\tau_{i}}\right), \forall \tau_{i}^{j} \in \boldsymbol{\tau}, \forall t \\
M\left(2-{ }^{t} A_{\tau_{i}^{j}}^{a}-H_{\tau_{i}}\right) \geq-U_{\tau_{i}^{j}}+{ }^{t} G_{\tau_{i}^{j}}^{a} \geq \\
M\left({ }^{t} A_{\tau_{i}^{j}}^{a}+H_{\tau_{i}}-2\right), \forall \tau_{i}^{j} \in \boldsymbol{\tau}, \forall t \\
\sum_{\tau_{i}^{j} \in \boldsymbol{\tau}}{ }^{t} G_{\tau_{i}^{j}}^{a} \leq C_{a}, \forall a \in A, \forall t \\
\sum_{r \in R}{ }^{t} R_{\tau_{i}^{j}}^{r} \geq 1-M\left(1-H_{\tau_{i}}\right), \forall \tau_{i}^{j} \in \boldsymbol{\tau}, \forall t \\
\sum_{\tau_{i}^{j} \in \boldsymbol{\tau}}{ }^{t} R_{\tau_{i}^{j}}^{r} \leq 1, \forall r \in R, \forall t \\
u b_{\tau_{i}^{j}} \geq f_{\tau_{i}^{j}}-s_{\tau_{i}^{j}} \geq l b_{\tau_{i}^{j}}, \forall \tau_{i}^{j} \in \boldsymbol{\tau} \\
s_{\tau_{x}^{y}}-f_{\tau_{i}^{j}} \geq W_{\left\langle\tau_{i}, \tau_{j}\right\rangle}, \forall \tau_{i}, \tau_{j} \in \boldsymbol{\tau} \mid, \forall W_{\left\langle\tau_{i}, \tau_{j}\right\rangle} \in \boldsymbol{T C} \\
f_{\tau_{x}^{y}}-s_{\tau_{i}^{j}} \leq D_{\left\langle\tau_{i}, \tau_{j}\right\rangle}^{r e l}, \forall \tau_{i}, \tau_{j} \in \boldsymbol{\tau} \mid \exists D_{\left\langle\tau_{i}, \tau_{j}\right\rangle}^{r e l} \in \boldsymbol{T C} \\
f_{\tau_{i}^{j}} \leq D_{\tau_{i}}^{a b s}, \forall \tau_{i} \in \boldsymbol{\tau} \mid \exists D_{\tau_{i}}^{a b s} \in \boldsymbol{T C}
\end{gathered}
$$

Equation 2 enforces that each subtask $\tau_{i}^{j}$ during each time epoch $[t, t+1)$ is assigned one agent. Equation 3 ensures that each subtask $\tau_{i}^{j}$ receives a sufficient portion of the effort of its assigned agent $a$ during time epoch $[t, t+1)$. Equation 4 ensures that agent $a$ is not oversubscribed. Equation 5 ensures that each subtask $\tau_{i}^{j}$ of each task $\tau_{i}$ that is to be completed (i.e., $H_{\tau_{i}}=1$ ) is assigned one resource $r$. Equation 6 ensures that each resource $r$ is assigned to only one subtask during each epoch $[t, t+1)$. Equation 7 requires the duration of subtask $\tau_{i}^{j}$ to be less than or equal to $u b_{\tau_{i}^{j}}$ and at least $l b_{\tau_{i}^{j}}$ units of time. Equation 8 requires that $\tau_{x}^{y}$ occurs at least $W_{\left\langle\tau_{i}^{j}, \tau_{x}^{y}\right\rangle}$ units of time after $\tau_{i}^{j}$. Equation 9 requires that the duration between the start of $\tau_{i}^{j}$ and the finish of $\tau_{x}^{y}$ is less than $D_{\left\langle\tau_{i}^{j}, \tau_{x}^{y}\right\rangle}^{r e l}$. Equation 10 requires that $\tau_{i}^{j}$ finishes before $D_{\tau_{i}^{j}}^{a b s}$ units of time have expired since the start of the schedule.

The stochasticity of the problem arises from the uncertainty in the upper and lowerbound of the durations $\left(u b_{\tau_{i}^{j}}, l b_{\tau_{i}^{j}}\right)$ of each of the steps in caring for a patient, the number and types of patients $\tau$ and the temporal constraints $\boldsymbol{T C}$ relating the start and finish of each step. These variables are a function of the resource and staff allocation variables ${ }^{t} R_{\tau_{i}^{j}}^{a},{ }^{t} A_{\tau_{i}^{j}}^{a}$, and patient task state $\Lambda_{\tau_{i}^{j}}$, which includes information on patient type (i.e. presenting with scheduled induction, scheduled 
cesarean section, or acute unplanned anomaly), gestational age, gravida, parity, membrane status, anesthesia status, cervix status, time of last exam and any co-morbidities. Formally, $\left(\left\{u b_{\tau_{i}^{j}}, l b_{\tau_{i}^{j}} \mid \tau_{i}^{j} \in \boldsymbol{\tau}\right\}, \boldsymbol{\tau}, \boldsymbol{T} \boldsymbol{C}\right) \sim P\left(\left\{{ }^{t} R_{\tau_{i}^{j}}^{a},{ }^{t} A_{\tau_{i}^{j}}^{a}, \Lambda_{\tau_{i}^{j}}, \forall t \in\right.\right.$ $[0,1, \ldots, T]\})$.

\section{A. The Role of the Resource Nurse}

The functions of a resource nurse are to assign nurses to take care of labor patients and to assign patients to labor beds, recovery room beds, operating rooms, ante-partum ward beds or post-partum ward beds. The resource nurse has substantial flexibility when assigning beds, and their decisions will depend upon the type of patient and the current status of the unit in question. They must also assign scrub technicians to assist with surgeries in operating rooms, and call in additional nurses if required. The corresponding decision variables for staff assignments and room/ward assignments in the above formulation are ${ }^{t} A_{\tau_{i}^{j}}^{a}$ and ${ }^{t} R_{\tau_{i}^{j}}^{r}$, respectively.

The resource nurse may accelerate, delay or cancel scheduled inductions or cesarean sections in the event that the floor is too busy. Resource nurses may also request expedited active management of a patient in labor. The decision variables for the timing of transitions between the various steps in the care process are described by $s_{\tau_{i}^{j}}$ and $f_{\tau_{i}^{j}}$. The commitments to a patient (or that patient's procedures) are represented by $H_{\tau_{i}}$.

The resource nurse may also reassign roles among nurses: For example, a resource nurse may pull a triage nurse or even care for patients herself if the floor is too busy. Or, if a patient's condition is particularly acute (e.g., the patient has severe pre-eclampsia), the resource nurse may assign one-to-one nursing. The level of attentional resources a patient requires and the level a nurse has available correspond to variables $U_{\tau_{i}^{j}}$ and ${ }^{t} G_{\tau_{i}^{j}}^{a}$, respectively. The resource nurse makes his or her decisions while considering current patient status $\Lambda_{\tau^{j}}$, which is manually transcribed on a whiteboard, shown in Figure 1.

\section{IMPLEMENTATION OF DECISION SUPPORT}

There are two fundamental challenges to providing decision support guidance through direct solution of the optimization problem depicted above. First, the computational complexity of the problem precludes production of real-time solutions. The computational complexity of satisfying constraints in Equations 2 10 is given by $O\left(2^{|A||R| T^{2}} C_{a}^{|A| T}\right)$, where $|A|$ is the number of agents, with each agent possessing an integer processing capacity of $C_{a}$; there are $n$ tasks $\tau_{i}$, each with $m_{i}$ subtasks; $|R|$ resources; and an integer-valued planning horizon of $T$ units of time. In practice, there are $\sim 10$ nurses (agents) who can care for up to two patients at a time (i.e., $C_{a}=2, \forall a \in A$ ), 20 different rooms (resources) of varying types, 20 patients (tasks) at any one time and a planning horizon of 12 hours or 720 minutes, yielding a worstcase complexity of $\sim 2^{10 * 20 * 720^{2}} 2^{10 * 720} \geq 2^{10^{6}}$, which is computationally intractable.

The second challenge to decision support guidance is that the precise form of the objective function (Equation 1) that resource nurses optimize for is unknown. Prior work has indicated that domain experts are adept at describing the features (high-level, contextual and task-specific) used in their decision making, yet it is more difficult for experts to describe how they reason about these features [10, 43]. As such, we applied a machine learning technique to learn a set of heuristic scheduling policies from demonstrations of resource nurse decision making. We then applied these learned policies to produce advice for the computer-based and robotic decision support systems.

\section{A. Learning from Resource Nurses}

In this section, we present a framework for learning (via expert demonstration) a set of heuristics for resource allocation and scheduling that emulates resource nurse decision making. For the purposes of our experiment, we focused on learning a policy for recommending which nurse should care for which patient, and for making patient room assignments. We demonstrate in Section VI that this technique produced highquality recommendations, as evidenced by an overall $90 \%$ accept rate of high-quality advice.

We applied action-driven learning rather than explicitly modeling a reward function and relying on dynamic programming or constraint solvers. This latter approach [3, 27, 36, 50, 55. 56] can quickly become computationally intractable for problems involving hundreds of tasks and tens of agents due to memory limitations. Approximate dynamic programming approaches exist that essentially reformulate the problem as regression [27, 32], yet the amount of data required to regress over a large state space remains challenging, and MDP-based task allocation and scheduling solutions exist only for simple problems [1, 51, 54].

Instead, we applied an apprenticeship scheduling algorithm [19] inspired by work in webpage ranking [22, 39]. The model representation, a graph with nodes and directed arcs, provides a suitable analogy for capturing the complex temporal dependencies (i.e., precedence, wait and deadline constraints) relating tasks within a scheduling problem. The approach uses pairwise comparisons between the actions taken (e.g., schedule agent $a$ to complete task $\tau_{i}$ at time $t$ ) and the set of actions not taken (e.g., unscheduled tasks at time $t$ ) to learn relevant model parameters and scheduling policies demonstrated by the training examples. This pairwise approach has the key advantage that it is nonparametric, in that the cardinality of the input vector is not dependent upon the number of tasks (or actions) that can be performed in any instance.

Consider a set of task-resource-agent $\left(\tau_{i}^{j}-R_{\tau_{i}^{j}}^{a}-A_{\tau_{i}^{j}}^{a}\right)$ assignments, denoted $\pi_{q} \in \Pi$. Each assignment $\pi_{q}$ has a set of associated features, $\gamma_{\pi_{q}}$, indicating patient type (i.e. presenting with scheduled induction, scheduled cesarean section, or acute unplanned anomaly), bed type, whether or not the bed is occupied, and staff status (i.e. number of patients for which the staff member is serving as primary nurse, covering nurse, baby nurse, or scrub technician). Next, consider a set of $m$ observations, $O=\left\{O_{1}, O_{2}, \ldots, O_{m}\right\}$. Each observation consists of a feature vector describing the task-resource-agent 
tuple $\pi_{q}$ scheduled by the expert demonstrator (including a null task $\tau_{\emptyset}$, resource $r_{\emptyset}$ or agent $a_{\emptyset}$ if no task, resource or agent was scheduled). The goal is to then learn a policy that correctly determines which task-resource-agent tuple $\pi_{q}$ to schedule as a function of feature state.

$$
\begin{gathered}
{ }^{\operatorname{rank}} \theta_{\left\langle\pi_{q}, \pi_{r}\right\rangle}^{m}:=\left[\gamma_{\pi_{q}}-\gamma_{\pi_{r}}\right], y_{\left\langle\pi_{q}, \pi_{r}\right\rangle}^{m}=1, \\
\forall \pi_{r} \in \Pi \backslash \pi_{q}, \forall O_{m} \in \boldsymbol{O} \mid \pi_{q} \text { scheduled in } O_{m} \\
{ }^{r a n k} \theta_{\left\langle\pi_{r}, \pi_{q}\right\rangle}^{m}:=\left[\gamma_{\pi_{r}}-\gamma_{\pi_{q}}\right], y_{\left\langle\pi_{r}, \pi_{q}\right\rangle}^{m}=0, \\
\forall \pi_{r} \in \Pi \backslash \pi_{q}, \forall O_{m} \in \boldsymbol{O} \mid \pi_{q} \text { scheduled in } O_{m} \\
\widehat{\pi_{q^{*}}}=\underset{\pi_{q} \in \Pi}{\operatorname{argmax}} \sum_{\pi_{q} \in \Pi} f_{\text {priority }}\left(\pi_{q}, \pi_{r}\right)
\end{gathered}
$$

In order to learn to correctly assign the subsequent task to the appropriate resource and/or agent, we transform each observation $O_{m}$ into a new set of observations by performing pairwise comparisons between the scheduled assignment $\pi_{q}$ and the set of assignments $s$ that were not scheduled (Equations 11/12). Equation 11 creates a positive example for each observation in which a $\pi_{q}$ was scheduled. This example consists of the input feature vector, $\phi_{\left\langle\pi_{q}, \pi_{r}\right\rangle}^{m}$, and a positive label, $y_{\left\langle\pi_{q}, \pi_{r}\right\rangle}^{m}=1$. Each element of the input feature vector $\phi_{\left\langle\pi_{q}, \pi_{r}\right\rangle}^{m}$ is computed as the difference between the corresponding values in the feature vectors $\gamma_{\pi_{q}}$ and $\gamma_{\pi_{r}}$, describing scheduled assignment $\pi_{q}$ and unscheduled task $\pi_{r}$. Equation 12 creates a set of negative examples with $y_{\left\langle\pi_{r}, \pi_{q}\right\rangle}^{m}=0$. For the input vector, we take the difference of the feature values between unscheduled assignment $\pi_{r}$ and scheduled assignment $\pi_{q}$.

We applied these observations to train a decision-tree classifier $f_{\text {priority }}\left(\pi_{q}, \pi_{r}\right) \in\{0,1\}$ to predict whether it is better to make the task-resource-agent assignment $\pi_{q}$ as the next assignment rather than $\pi_{r}$. Given this pairwise classifier, we can determine which single assignment $\pi_{q}{ }^{*}$ is the highest-priority assignment according to Equation 13 by determining which assignment is most often of higher priority in comparison to the other assignments in $\Pi$.

In our experiments, $f_{\text {priority }}\left(\pi_{q}, \pi_{r}\right)$ was applied directly to generate high-quality recommendations. We generated lowquality advice using two methods: The first method recommended the action that minimized Equation 13, instead of maximizing it. This approach would typically generate infeasible advice (e.g., move a patient to a room that is currently occupied). A second method was applied to offer low-quality but feasible advice (e.g., assign a post-operating patient to triage). This was achieved by evaluating Equation 13 after filtering the space of possible actions to include only feasible actions (per the constraints in Equations 2. 10. Recommendations for the low-quality condition were produced by randomly selecting between these two methods in order to mitigate ordering effects.

The dataset used for training was generated by seven

\begin{tabular}{|c|c|c|c|}
\hline \multirow{2}{*}{\multicolumn{2}{|c|}{ Robotic Decision Support }} & \multicolumn{2}{|c|}{ Response } \\
\hline & & Accept & Reject \\
\hline \multirow{2}{*}{ Advice Quality } & High & $130(44.5 \%)$ & $16(5.48 \%)$ \\
\hline & Low & $16(5.48 \%)$ & $130(44.5 \%)$ \\
\hline \multirow{2}{*}{\multicolumn{2}{|c|}{ Virtual Decision Support }} & \multicolumn{2}{|c|}{ Response } \\
\hline & & Accept & Reject \\
\hline \multirow{2}{*}{ Advice Quality } & High & $134(45.3 \%)$ & $14(4.78 \%)$ \\
\hline & Low & $19(6.48 \%)$ & $126(43.0 \%)$ \\
\hline
\end{tabular}
resource nurses working with the simulation for a total of $2^{1 / 2}$
TABLE I

CONFUSION MATRIX FOR PARTICIPANTS SHOWN AS A RAW COUNT AND PERCENTAGE OF THE WHOLE.

TABLE II

CORRECT ACCEPT AND REJECT DECISIONS MADE WITH COMPUTER-BASED (C-ACCEPT, C-REJECT) VERSUS ROBOTIC (R-ACCEPT, R-REJECT) DECISION SUPPORT, AS A FUNCTION OF TRIAL NUMBER, SHOWN AS A RAW COUNT AND PERCENTAGE OF THE WHOLE.

\begin{tabular}{|c|c|c|c|c|}
\cline { 2 - 5 } \multicolumn{1}{c|}{} & \multicolumn{4}{c|}{ Trial Number } \\
\cline { 2 - 5 } \multicolumn{1}{c|}{} & \multicolumn{3}{c|}{ Bad Advice } & \multicolumn{2}{c|}{ Good Advice } \\
\cline { 2 - 5 } \multicolumn{1}{c|}{} & 1 & 2 & 3 & 4 \\
\hline C-Accept & $5(10.4 \%)$ & $4(6.7 \%)$ & $41(82.0 \%)$ & $49(92.5 \%)$ \\
\hline R-Accept & $9(17.6 \%)$ & $5(9.6 \%)$ & $43(91.5 \%)$ & $44(93.6 \%)$ \\
\hline
\end{tabular}

\begin{tabular}{|c|c|c|c|c|}
\cline { 2 - 5 } \multicolumn{1}{c|}{} & \multicolumn{4}{c|}{ Trial Number } \\
\cline { 2 - 5 } \multicolumn{1}{c|}{} & \multicolumn{3}{|c|}{ Good Advice } & \multicolumn{2}{c|}{ Bad Advice } \\
\cline { 2 - 5 } \multicolumn{1}{c|}{} & 1 & 2 & 3 & 4 \\
\hline C-Reject & $2(28.6 \%)$ & $1(2.8 \%)$ & $11(73.3 \%)$ & $20(87.0 \%)$ \\
\hline R-Reject & $3(8.6 \%)$ & $1(10.0 \%)$ & $21(84.0 \%)$ & $16(94.1 \%)$ \\
\hline
\end{tabular}

hours, simulating 60 hours of elapsed time on a real labor floor. This yielded a dataset of more than 3,013 individual decisions. None of the seven resource nurses who contributed to the dataset participated in the experiment.

\section{RESUlts}

We report statistical testing of our hypotheses here. We defined statistical significance at the $\alpha=0.05$ level.

\section{A. Analysis \& Discussion of $\boldsymbol{H} \boldsymbol{1}$}

Objective measures of compliance and reliance were assessed based on the participant's "accept" or "reject" responses to each decision support recommendation. Statistics on hits, misses, false alarms and correct rejections are shown in Table [1. Results from a z-test for two proportions indicated no statistically significant difference in the Type II error rates for the robotic $\left(p_{R}=13.1 \%\right)$ and computer-based $\left(p_{C}=11.0 \%\right)$ decision support conditions $(z=0.562, p=0.713)$, nor in the rates of correct "accept" responses to high-quality advice $\left(p_{R}=90.5 \%, p_{C}=89.0 \%, p=0.713\right)$ and "reject" responses to low-quality advice ( $p_{R}=86.9 \%, p_{C}=89.0 \%, p=0.287$ ) across the two conditions. Results from a TOST equivalence test using two z-tests for two proportions indicated that the rates of error, appropriate compliance and appropriate reliance between the robotic and virtual decision support conditions were equivalent within $95 \%$ confidence.

We also analyzed the rates of Type I and Type II errors in the second and third trials, at the transition in advice quality (Table III). Fisher's exact test found a significant difference 
TABLE III

SubJective Measures Post-Trial Questionnaire With STATISTICAL SIGNIFICANCE. QUESTIONS 1-5 WERE RESPONDED TO ON A 7-POINT SCALE, AND QUESTIONS 6-9 ON A 10-POINT SCALE.

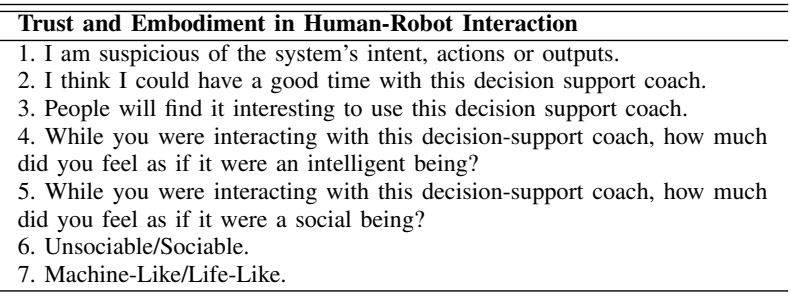

in the rate of incorrect "accept" of low quality advice (Type I error) across the second and third trials for the computer-based decision support ( $6.7 \%$ vs. $26.7 \%, p=0.046)$, but not for the robotic support $(9.6 \%$ vs. $16.0 \%, p=0.461)$. A significant difference was also found in the rate of incorrect "reject" of high-quality advice (Type II error) across the second and third trials for the computer-based decision support $(2.8 \%$ vs. $18.0 \%, p=0.040$ ), but not for robotic decision support ( $10.0 \%$ vs. $8.5 \%, p \sim 1.0)$. In other words, participants' rate of Type I error associated with computer-based support increased significantly when participants had received highquality advice in the previous trial. Similarly, the rate of Type II error associated with computer-based support increased significantly when participants had received low-quality advice in the previous trial. No such significant differences were found for the robotic support conditions.

H1 Takeaway: These results support H1, in that Type I and Type II error rates were comparable between robotic and computer-based decision support. Furthermore, embodiment appeared to offer performance gains, as indicated by lower error rates after the quality of recommendation changed midexperiment. These are encouraging findings because they provide evidence that a robotic assistant may be able to participate in decision making with nurses without eliciting inappropriate dependence. One potential rationale for these results is that experts may be less susceptible to the negative effects of embodiment, as has been documented for experienced users interacting with anthropomorphic agents [40]. We note that our study was conducted with a stationary robot, in which movement was limited to co-speech gestures. Further investigation is warranted for situations in which experts interact with mobile service robots that participate in decision-making.

\section{B. Analysis \& Discussion of $\boldsymbol{H} 2$}

A composite measure of trust was computed, as in [21]. Results from a repeated-measures ANOVA (RANOVA) demonstrated a statistically significant increase in the average rating for the decision support system under the high-quality advice condition ( $M=5.39, S D=0.666)$ as compared with the low-quality condition $(M=3.49, S D=1.26)(F(1,14)=$ $46.3, p<0.001)$. However, a RANOVA yielded no statistically significant difference in trust between the robotic $(M=4.41$, $S D=1.32)$ and computer-based $(M=4.48, S D=1.47)$ embodiment conditions $(F(1,14)=0.450, p=0.513)$. Results from a TOST equivalence test, using two t-tests, indicated that subjects' trust ratings for the computer-based and robotic support were within one point of one another on a 7-point Likert Scale.

We observed significant differences in the attitudinal assessment of the robotic versus computer-based decision support conditions for Questions 2, 3, 5, 6 in Table III. indicating that participants rated the robotic system more favorably. The result was established using a two-way omnibus Friedman test, followed by pairwise Friedman tests. The test statistics for the pairwise Friedman tests were $p=0.028,0.007,0.043$, and 0.005 , respectively. Strikingly, there was not a single question (out of 37) for which participants rated the computer-based decision support significantly better than the robotic support.

We also found that the subjective perception of the character of the robot was significantly less sensitive to transitions in advice quality than the computer-based decision support. We computed the frequency with which the ratings of one embodiment condition subsumed the other, and vice versa. Specifically, we defined $x_{R, L}$ as the Likertscale rating for a given question and a particular participant in the robotic low-quality advice condition, and likewise for the high-quality condition, $x_{R, H}$. The variables $x_{C, L}$, $x_{C, H}$ were similarly defined for the computer-based lowand high-quality conditions. The robotic condition was defined as subsuming the computer-based condition if either $\min \left(x_{R, L}, x_{R, H}\right) \leq \min \left(x_{C, L}, x_{C, H}\right) \leq \max \left(x_{C, L}, x_{C, H}\right)<$ $\max \left(x_{R, L}, x_{R, H}\right)$ or $\min \left(x_{R, L}, x_{R, H}\right)<\min \left(x_{C, L}, x_{C, H}\right) \leq$ $\max \left(x_{C, L}, x_{C, H}\right) \leq \max \left(x_{R, L}, x_{R, H}\right)$, and vice versa for the computer-based condition subsuming the robotic condition. A $\chi^{2}$ test indicated that the participants' subjective evaluation according to Questions 1, 4, 6, 7 ( $p=0.045,0.022,0.005$ and 0.0043 , respectively) changed more significantly under the computer-based condition than the robotic condition. There were no questions for which the response changed more significantly under the robotic condition versus the computerbased condition. In other words, the subjective assessment of the robot was more robust to advice quality changes than the computer-based decision support. Further investigation is warranted to determine whether these effects persist over time as the users habituate to interaction with the robot.

H2 Takeaway: Our findings support $\mathrm{H} 2$ in that the robotic system was rated more favorably on attitudinal assessment than computer-based decision support, even as it engendered appropriate dependence. It is inevitable that a service robot will occasionally make poor-quality suggestions, and we positively note that the robot engendered greater tolerance of errors than the computer-based decision support. These results indicate a positive signal for successful adoption of a robot that participates in a resource nurse's decision making.

\section{Pilot Demonstration of a Robotic Assistant ON THE LABOR AND DELIVERY FLOOR}

Based on the positive results of our experiment, we conducted a pilot demonstration in which a robot assisted resource 


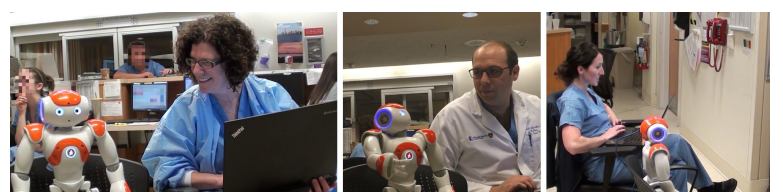

Fig. 3. Images of the robot system in action on the labor floor.

nurses on a labor and delivery floor at a tertiary care center.

\section{A. Robot System Architecture}

The system was comprised of subsystems providing the vision, communication and decision support capabilities.

Vision System: In our experiments, the statuses of patients, nurses and beds were provided and updated in the simulation. In contrast, nurses and robots on a real labor floor must read handwritten information off of a whiteboard (i.e., "dashboard") depicted in Figure 11. Extracting and parsing this information autonomously with high accuracy and reliability presents a substantial technical challenge. We make two assumptions to address this: (1) that the set of physician and nurse names is closed and known in advance, and (2) that patient names are transcribed for the robot upon patient arrival.

In our demonstration, we leveraged the structured nature of the dashboard to introduce priors that ensured patient information was interpretable. Rows on the dashboard indicate room assignments, while columns indicate patient parameters (e.g., attending physician, gestational age, etc.). Once our robot captured an image of the dashboard on the labor and delivery floor, we applied a Canny edge detection operator [8] and Hough transformation [18] to isolate the handwriting in individual grid cells. The contents of each grid cell were processed using a classification technique appropriate to the data type therein. Numeric fields were parsed using a Convolutional Neural Network $(\mathrm{CNN})^{1}$ trained on MNIST data, while alphabetical fields with known sets of possible values (e.g. attending physician, nurse names) were parsed using a multi-class $\mathrm{CNN}$ trained on handwriting ${ }^{2}$

Handwriting samples (28 uniquely written alphabets) were used as a basis for generating classifier training data. Fonts were created from the provided samples and used (along with system fonts) to create a large set of binary images containing samples of nurse names. These synthetic writing samples were constructed with a range of applied translations, scalings, and kerning values within a 75×30 pixel area.

The vision system was used to determine the current status of patient-nurse allocations, nurse role information and room usage. Prior to deployment, we performed a validation of the vision system and found our recognition system to correctly classify handwritten samples across 15 classes (names) with $\sim 83.7 \%$ overall accuracy and $97.8 \%$ average accuracy. These

\footnotetext{
${ }^{1}$ Thanks to Mikhail Sirontenko for developing this package, which is available at https://sites.google.com/site/mihailsirotenko/projects/cuda-cnn

${ }^{2}$ We utilize a network with a the following architecture:

$75 \times 30$ input layer $\rightarrow 5 \times 5$ kernel convolution layer $\rightarrow 2 \times 2$ kernel maxpool layer $\rightarrow 5 \times 5$ kernel convolution layer $\rightarrow 2 \times 2$ kernel maxpool layer $\rightarrow 100$ node dense layer $\rightarrow$ classification layer.
}

results were obtained without performing any environmental manipulations (adjusting lighting, using high-resolution cameras, etc.). In the pilot deployment, our vision system assisted humans with transcription of patient data.

Communication: CMUSphinx [2] was employed for robot speech recognition. To achieve high performance in a live setting, we defined a list of template-based phrases a user might utter, such as "Where should I move the patient in room [\#]?" or "Who should nurse [Name] take care of?" All possible instantiations were enumerated based on information available a priori (e.g., the list of nurse names). Levenshtein distance [30] was computed to infer the phrase most likely uttered by the speaker, and the appropriate corresponding query was issued to the decision support system.

Decision Support: The live pilot demonstration of the robot used the same mechanism for generating decision support as that used during our experiments. However, unlike the experiments, the decision support system's input was taken from the vision subsystem, and the user query from the communication subsystem. The set of possible actions to be recommended was filtered according to the query as recognized by the communication subsystem. For example, if the user asked, "Where should I move the patient in room 1A?," actions that would change nurse assignments were not considered. The recommended action was communicated to the user via text-to-speech software.

Feedback from Nurses and Physicians: We conducted a test demonstration on the labor floor (Figure 3). Three users interacted with the robot over the course of three hours. Ten queries were posed to the robot; seven resulted in successful exchanges and three failed due to background noise. A live recording of the demo can be seen at http://tiny.cc/RobotDemo. After interacting with the robotic support, User 1, a physician, said "I think the [robot] would allow for a more even dispersion of the workload amongst the nurses. In some hospitals...more junior nurses were given the next patient...more senior nurses were allowed to only have one patient as opposed to two." User 2, a resource nurse said, "New nurses may not understand the constraints and complexities of the role, and I think the robot could help give her an algorithm ... that she can practice, repeat, and become familiar with so that it becomes second nature to her." User 3, a labor nurse offered, "I think you could use this robot as an educational tool."

\section{CONCLUSION}

This paper addresses two barriers to fielding intelligent hospital service robots that take initiative to participate with nurses in decision making. We find experimental evidence that experts performing decision making tasks may be less susceptible to the negative effects of support embodiment. Further our decision support was able to produce context-specific decision strategies and apply them to make reasonable suggestions for which tasks to perform and when. Finally, based on the previous two findings, we conducted a first successful test demonstration in which a robot assisted resource nurses on a labor and delivery floor in a tertiary care center. 


\section{REFERENCES}

[1] A novel multi-agent reinforcement learning approach for job scheduling in grid computing. Future Generation Computer Systems, 27(5):430 - 439, 2011.

[2] Cmu sphinx open source speech recognition toolkit, January 2016. URL http://cmusphinx.sourceforge.net/.

[3] Pieter Abbeel and Andrew Y. Ng. Apprenticeship learning via inverse reinforcement learning. In ICML. ACM, 2004. ISBN 1-58113-838-5. doi: 10.1145/1015330. 1015430. URL http://doi.acm.org/10.1145/1015330. 1015430

[4] Wilma A Bainbridge, Justin W Hart, Elizabeth S Kim, and Brian Scassellati. The benefits of interactions with physically present robots over video-displayed agents. International Journal of Social Robotics, 3(1):41-52, 2011.

[5] D. Bertsimas and R. Weismantel. Optimization over Integers. Dynamic Ideas, 2005.

[6] Richard Bloss. Mobile hospital robots cure numerous logistic needs. Industrial Robot: An International Journal, 38(6):567-571, 2011.

[7] L. Brandenburg, P. Gabow, G. Steele, J. Toussaint, and B. J. Tyson. Innovation and best practices in health care scheduling. Technical report, 2015.

[8] John Canny. A computational approach to edge detection. IEEE Transactions on Pattern Analysis and Machine Intelligence, (6):679-698, 1986.

[9] Jessie YC Chen, Michael J Barnes, and Michelle HarperSciarini. Supervisory control of multiple robots: Humanperformance issues and user-interface design. Systems, Man, and Cybernetics, Part C: Applications and Reviews, IEEE Transactions on, 41(4):435-454, 2011.

[10] Tsang-Hsiang Cheng, Chih-Ping Wei, and Vincent S. Tseng. Feature selection for medical data mining: Comparisons of expert judgment and automatic approaches. In Proc. CBMS, pages 165-170, 2006.

[11] Mary L Cummings and Stephanie Guerlain. Developing operator capacity estimates for supervisory control of autonomous vehicles. Human Factors: The Journal of the Human Factors and Ergonomics Society, 49(1):1-15, 2007.

[12] E. J. de Visser, F. Krueger, P. McKnight, S. Scheid, M. Smith, S. Chalk, and R. Parasuraman. The world is not enough: Trust in cognitive agents. Proceedings of the Human Factors and Ergonomics Society Annual Meeting, 56(1):263-267, 2012.

[13] Munjal Desai, Mikhail Medvedev, Marynel Vázquez, Sean McSheehy, Sofia Gadea-Omelchenko, Christian Bruggeman, Aaron Steinfeld, and Holly Yanco. Effects of changing reliability on trust of robot systems. In Human-Robot Interaction (HRI), 2012 7th ACM/IEEE International Conference on, pages 73-80. IEEE, 2012.

[14] Munjal Desai, Poornima Kaniarasu, Mikhail Medvedev, Aaron Steinfeld, and Holly Yanco. Impact of robot failures and feedback on real-time trust. In Proceed- ings of the 8th ACM/IEEE International Conference on Human-robot Interaction, HRI '13, pages 251-258, Piscataway, NJ, USA, 2013. IEEE Press. ISBN 9781-4673-3055-8. URL http://dl.acm.org/citation.cfm?id= 2447556.2447663

[15] Nicole DiGiose. Hospitals hiring robots, February 2013. URL http://www.electronicproducts.com/Computer Peripherals/Systems/Hospitals_hiring_robots.aspx

[16] R. K. Dismukes, B. A. Berman, and L. D. Loukopoulous. The Limits of Expertise: Rethinking Pilot Error and the Causes of Airline Accidents. Ashgate Publishing, 2007.

[17] Stephen R Dixon and Christopher D Wickens. Automation reliability in unmanned aerial vehicle control: A reliance-compliance model of automation dependence in high workload. Human Factors: The Journal of the Human Factors and Ergonomics Society, 48(3):474-486, 2006.

[18] Richard O Duda and Peter E Hart. Use of the hough transformation to detect lines and curves in pictures. Communications of the ACM, 15(1):11-15, 1972.

[19] Matthew Gombolay, Reed Jensen, Jessica Stigile, SungHyun Son, and Julie Shah. Apprenticeship scheduling: Learning to schedule from human experts. In Proceedings of the International Joint Conference on Artificial Intelligence (IJCAI), New York City, NY, U.S.A., July 9-15 2016.

[20] John Hu, Aaron Edsinger, Yi-Je Lim, Nick Donaldson, Mario Solano, Aaron Solochek, and Ronald Marchessault. An advanced medical robotic system augmenting healthcare capabilities-robotic nursing assistant. In Robotics and Automation (ICRA), 2011 IEEE International Conference on, pages 6264-6269. IEEE, 2011.

[21] J.-Y. Jian, A. M. Bisantz, and C. G. Drury. Foundations for an empirically determined scale of trust in automated systems. International Journal of Cognitive Ergonomics, 4(1):53-71, 2000.

[22] Rong Jin, Hamed Valizadegan, and Hang Li. Ranking refinement and its application to information retrieval. In Proceedings of the 17th International Conference on World Wide Web, pages 397-406. ACM, 2008. ISBN 978-1-60558-085-2. doi: 10.1145/1367497.1367552. URL http://doi.acm.org/10.1145/1367497.1367552.

[23] David B Kaber and Mica R Endsley. Out-of-the-loop performance problems and the use of intermediate levels of automation for improved control system functioning and safety. Process Safety Progress, 16(3):126-131, 1997.

[24] S. M. Kehle, N. Greer, I. Rutks, and T. Wilt. Interventions to improve veterans' access to care: A systematic review of the literature. Journal of General Internal Medicine, 26(2):689-696, 2011.

[25] Cory D Kidd and Cynthia Breazeal. Effect of a robot on user perceptions. In Intelligent Robots and Systems, 2004.(IROS 2004). Proceedings. 2004 IEEE/RSJ International Conference on, volume 4, pages 3559-3564. IEEE, 2004. 
[26] Sara Kiesler, Aaron Powers, Susan R Fussell, and Cristen Torrey. Anthropomorphic interactions with a robot and robot-like agent. Social Cognition, 26(2):169-181, 2008.

[27] G. Konidaris, S. Osentoski, and P. Thomas. Value function approximation in reinforcement learning using the fourier basis. In Proc. AAAI, pages 380-385, 2011.

[28] John D. Lee and Katrina A. See. Trust in automation: Designing for appropriate reliance. Human Factors, 46 (1):50-80, 2004.

[29] K. W. Lee, W. Peng, S.-A. Jin, and C. Yan. Can robots manifest personality?: An empirical test of personality recognition, social responses, and social presence in humanrobot interaction. Journal of Communication, 56 (4):754-772, 2006.

[30] Vladimir I Levenshtein. Binary codes capable of correcting deletions, insertions, and reversals. In Soviet physics doklady, volume 10, pages 707-710, 1966.

[31] Daniel Leyzberg, Samuel Spaulding, and Brian Scassellati. Personalizing robot tutors to individuals' learning differences. In Proceedings of the 2014 ACM/IEEE international conference on Human-robot interaction, pages 423-430. ACM, 2014.

[32] V. Mnih, K. Kavukcuoglu, D. Silver, A. A. Rusu, J. Veness, M G. Bellemare, A. Graves, M. Riedmiller, A. K. Fidjeland, G. Ostrovski, S. Petersen, C. Beattie, A. Sadik, I. Antonoglou, H. King, D. Kumaran, D. Wierstra, S. Legg, and D. Hassabis. Human-level control through deep reinforcement learning. Nature, 518(7540):529533, 2015.

[33] Ryota Murai, Tadashi Sakai, Yuma Honda, et al. Recognition of $3 \mathrm{~d}$ dynamic environments for mobile robot by selective memory intake and release of data from $2 \mathrm{~d}$ sensors. In System Integration (SII), 2012 IEEE/SICE International Symposium on, pages 621-628. IEEE, 2012.

[34] Ryota Murai, Tadashi Sakai, Hiroyuki Kawano, Yoshihiko Matsukawa, Yuma Honda, KC Campbell, et al. A novel visible light communication system for enhanced control of autonomous delivery robots in a hospital. In System Integration (SII), 2012 IEEE/SICE International Symposium on, pages 510-516. IEEE, 2012.

[35] Bilge Mutlu and Jodi Forlizzi. Robots in organizations: the role of workflow, social, and environmental factors in human-robot interaction. In Human-Robot Interaction (HRI), 2008 3rd ACM/IEEE International Conference on, pages 287-294. IEEE, 2008.

[36] P. Odom and S. Natarajan. Active advice seeking for inverse reinforcement learning. In Proceedings of AAAI, pages 4186-4187, 2015.

[37] Dan R Olsen Jr and Stephen Bart Wood. Fan-out: measuring human control of multiple robots. In Proceedings of the SIGCHI conference on Human factors in computing systems, pages 231-238. ACM, 2004.

[38] Ali Gürcan Özkil, Zhun Fan, Steen Dawids, H Aanes, Jens Klæstrup Kristensen, and Kim Hardam Christensen. Service robots for hospitals: A case study of transportation tasks in a hospital. In Automation and Logistics,
2009. ICAL'09. IEEE International Conference on, pages 289-294. IEEE, 2009.

[39] Tapio Pahikkala, Evgeni Tsivtsivadze, Antti Airola, Jorma Boberg, and Tapio Salakoski. Learning to rank with pairwise regularized least-squares. In SIGIR 2007 Workshop on Learning to Rank for Information Retrieval, pages 27-33, 2007.

[40] R. Pak, N. Fink, M. Price, B. Bass, and L. Sturre. Decision support aids with anthropomorphic characteristics influence trust and performance in younger and older adults. Ergonomics, 55(9):1059-1072, 2012.

[41] R. Parasuraman, T.B. Sheridan, and Christopher D. Wickens. A model for types and levels of human interaction with automation. Trans. SMC-A, 30(3):286-297, 2000.

[42] S. D. Pizer and J. C. Prentice. What are the consequences of waiting for health care in the veteran population? Journal of General Internal Medicine, 26(2):676-682, 2011.

[43] Hema Raghavan, Omid Madani, and Rosie Jones. Active learning with feedback on features and instances. Journal of Machine Learning Research, 7:1655-1686, December 2006. ISSN 1532-4435.

[44] P. Robinette, A. M. Howard, and A. R. Wagner. Overtrust of robots in emergency evacuation scenarios. In Proc. HRI, 2016.

[45] Marc Schröder and Jürgen Trouvain. The german textto-speech synthesis system mary: A tool for research, development and teaching. International Journal of Speech Technology, 6(4):365-377, 2003.

[46] Thomas B Sheridan. Adaptive automation, level of automation, allocation authority, supervisory control, and adaptive control: Distinctions and modes of adaptation. Systems, Man and Cybernetics, Part A: Systems and Humans, IEEE Transactions on, 41(4):662-667, 2011.

[47] S. A. Shipman and C. A. Sinsky. Expanding primary care capacity by reducing waste and improving efficiency of care. Health Affairs, 32(11):1990-1997, 2013.

[48] Leila Takayama and Caroline Pantofaru. Influences on proxemic behaviors in human-robot interaction. In Intelligent Robots and Systems, 2009. IROS 2009. IEEE/RSJ International Conference on, pages 5495-5502. IEEE, 2009.

[49] Adriana Tapus, Cristian Tapus, and Maja Matarić. The role of physical embodiment of a therapist robot for individuals with cognitive impairments. In Robot and Human Interactive Communication, 2009. RO-MAN 2009. The 18th IEEE International Symposium on, pages 103-107. IEEE, 2009.

[50] Adam Vogel, Deepak Ramach, Rakesh Gupta, and Antoine Raux. Improving hybrid vehicle fuel efficiency using inverse reinforcement learning. In Proceedings of AAAI, pages 384-390, 2012.

[51] Y.-C. Wang and J. M. Usher. Application of reinforcement learning for agent-based production scheduling. Eng. Appl. Artif. Intell., 18(1):73-82, 2005.

[52] C. D. Wickens, J. G. Hollands, S. Banbury, and R. Para- 
suraman. Engineering psychology and human performance. Pearson Education, 2013.

[53] Christopher D Wickens, Huiyang Li, Amy Santamaria, Angelia Sebok, and Nadine B Sarter. Stages and levels of automation: An integrated meta-analysis. In Proceedings of the Human Factors and Ergonomics Society Annual Meeting, volume 54, pages 389-393. SAGE Publications, 2010.

[54] W. Zhang and T. G. Dietterich. A reinforcement learning approach to job-shop scheduling. In Proc. IJCAI, pages 1114-1120. Morgan Kaufmann, 1995.

[55] Jiangchuan Zheng, Siyuan Liu, and Lionel Ni. Robust bayesian inverse reinforcement learning with sparse behavior noise. In Proceedings of AAAI, pages 2198-2205, 2015.

[56] Brian D. Ziebart, Andrew Maas, J. Andrew Bagnell, and Anind K. Dey. Maximum entropy inverse reinforcement learning. In Proceedings of AAAI, pages 1433-1438, 2008. ISBN 978-1-57735-368-3. URL http://dl.acm.org/ citation.cfm?id=1620270.1620297. 